\title{
Monte Carlo Simulations of the Spatial Transport of Excitons in a Quantum Well Structure
}

\author{
Yutaka Takahashi* \\ Department of Electrical and Information Engineering, Yamagata University, Jonan, \\ Yonezawa-shi, Yamagata 992, Japan
}

(October 17, 2018)

\begin{abstract}
The in-plane spatial transport of nonequilibrium excitons in a GaAs quantum well structure has been simulated with the ensemble Monte Carlo method. The simulation has been performed for excitons in the presence of residual heavy holes including the interparticle Coulomb scatterings, LA phonon scatterings, and exciton/carrier-interface roughness scatterings. It has been found that, in contrast to the free electrons/holes system in which the carrier-carrier scattering is significant, the interface roughness scattering is the dominant process for excitons because of the relatively small scattering rate of excitoncarrier and exciton-exciton scatterings. This strongly affects both the spatial motion and the energy relaxation of excitons. The spatial and momentum distributions of excitons have been simulated up to 500 ps at several exciton temperatures and interface roughness parameters. We have found that the exciton transport can be regarded as a diffusive motion whose diffusion coefficient varies with time. The diffusivity varies because the average velocity of excitons change through the energy transfer between the excitons and the
\end{abstract}


residual heavy hole/lattice system.

07.05.Tp,02.70.Lq, 73.50.-h, 73.61.Ey, 78.66.-w

Typeset using REVTEX 


\section{INTRODUCTION}

In-plane transport of photogenerated electrons/holes or excitons confined in 2dimensional (2D) space of quantum well (QW) structures has attracted much attention as it contains rich physics not accessible in the stationary transport of conduction electrons. It is also important from the practical point of view since the spatial resolutions of position sensitive optical measurements such as the photoluminescence (PL) via optical microscopes and the recently-developed near-field scanning optical microscopes (NSOM) $\frac{1}{1}$ are determined by the diffusion length of photocarriers.

The measurements of photocarrier transport, which is a transient phenomenon, are difficult because it requires high spatial and temporal resolutions. There have been several attempts to measure it in QW's of GaAs/AlGaAs with various techniques under different experimental conditions. The exciton localization was reported by Hegarty et al $\mathrm{l}$ using the

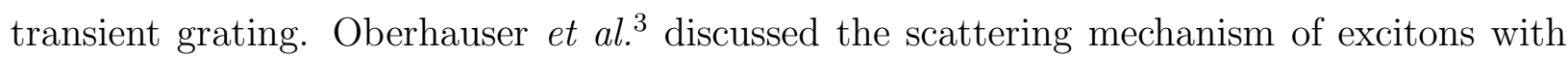
the same method. The pump-probe technique was utilized by Smith et al. to investigate the excitation density dependence of the spatial motion. Tsen et al. the time and spatially resolved Raman spectroscopy and discussed the spatial transport as well as the energy relaxation. The time-of-flight (tof) method with a photomask was developed by Hillmer et al $\mathrm{B}$ and was applied to study the temperature and excitation energy dependence of the transport in QW's under low excitation conditions. 212 The similar tof

method with an optical fiber was adopted by Akiyama et al. 13 who reported the transport properties in the wide range of excitation density in doped and nondoped QW's.

In spite of these experimental efforts the physical mechanism of transport is not fully understood in the microscopic level so far. This is because the spatial transport of photogenerated carriers is closely related with the longitudinal relaxation of carriers in the momentum space (i.e. the energy relaxation). This makes the problem further complicated as compared with the transport of conduction electrons for which the momentum space distribution is stationary. A distribution function of photocarriers, which are initially generated nonther- 
mally at some point in the momentum space, changes gradually due to the scatterings of carriers, and approaches the thermal equilibrium. At the same time carriers spread spatially from the point where they are initially generated. Since the scattering processes are affected both by the momentum distribution and the spatial distribution (or the density) of carriers and the carrier velocities are determined by the momentum distribution, the time evolutions of the momentum and the spatial distribution are mutually dependent. Thus it is necessary to solve the time evolution of momentum and spatial distribution simultaneously to understand the transport properties of photogenerated carriers. Several authors 8 reported the theoretical studies on the spatial transport of excitons in QW's, but they treated the momentum distribution in thermal equilibrium, leaving its dynamic evolution out of account.

There are several approaches to the theoretical analysis of the longitudinal momentum relaxation (energy relaxation) of photogenerated carriers:15 Analytical estimations based on Green's functions, numerical integrations of Boltzmann equations, and numerical simulations such as ensemble Monte Carlo (EMC) method. The last method is essentially the same as solving (nonlinear) Boltzmann equations but, with the advent of fast computers, it has become widely used because it is possible to perform quantitative calculations for nonequilibrium distributions of carriers and easy to incorporate microscopic scattering mechanisms into the model. Another advantage of this method is that the realistic experimental conditions such as the shape of the sample, the initial, and the boundary conditions, can be used in the model calculations, allowing one to compare the theory and the experiment directly. (The details of this method is reviewed in Ref. 16.)

There are many EMC studies on the nonequilibrium dynamics of photogenerated carriers 24 both in bulk and QW structures. But this method simulates the dynamics of particles in momentum space and does not yield the information in real space. Another class of numerical simulation methods is the Molecular Dynamics (MD) approach, in which all particle trajectories are calculated classically in real space. Thus it naturally enables one to trace the motion of particles in real space. MD has been applied to the dynamics of 
nonequilibrium carriers recently by several authors, 25 , 26 however, when the number of participating particles grows, it becomes time-consuming and requires huge computer resources. An alternative method is required to practically perform the numerical simulation in the long time scale.

In this paper the spatial transport and the momentum relaxation of excitons is numerically simulated. The spatial motion is treated by dividing the simulation area by mesh (concentric regions in the present case), and the dynamics in the momentum space is calculated in each region by EMC method at the density and the momentum distribution averaged in the region. The position and the momentum of the particle, and thus the density and the momentum distribution in each region are updated at every time step. This approximation allows one to trace the spatial distribution of particles at the spatial resolution comparable to the region size without the expense of fast computational speed of EMC method. The scattering mechanisms as carrier-carrier, exciton-exciton, exciton-carrier, carrier-LA phonon, exciton-LA phonon, carrier-interface roughness, and exciton-interface roughness scatterings are included in the simulations.

This paper is organized as follows: In Sec. II the simulation conditions are briefly described, followed by the detailed description the screening and scattering mechanisms, and the method of EMC simulation. In Sec. III $A$ the comparison of free $e /$ hh and exciton relaxation is discussed and in Sec. III $B$ the numerical results of the exciton spatial transport are given. They are discussed in comparison with the previous experimental works. Conclusions are given in Sec. IV.

\section{MODEL}

\section{A. Simulation Conditions}

The in-plane spatial motion and the energy relaxation of the nonequilibrium excitons are calculated in 2D space of nondoped QW structures of GaAs, with the well width $L_{z}=10 \mathrm{~nm}$. 
The infinite barrier height is assumed throughout. The simulations have been performed in a circular area of radius $10 \mu \mathrm{m}$. The initial excitons are spatially distributed in the Gaussian shape, $C \exp \left(-4 \ln 2 \frac{x^{2}}{F W H M^{2}}\right)$, with its full width at half maximum (FWHM) $6 \mu \mathrm{m}$, and their momentum distribution is in the Bose distribution (which is essentially identical to the Boltzmann distribution at the density we have used), where the exciton temperature $L_{e x}$ is used as a parameter for the initial momentum distributions. The peak areal density of excitons is $1.9 \times 10^{9} \mathrm{~cm}^{-2}$ at the center of the simulation area. The residual carriers (heavy holes) of uniform areal density $1 \times 10^{9} \mathrm{~cm}^{-2}$ is assumed over the whole simulation area as with Ref. 19, whose initial momentum distribution is in the Fermi distribution whose temperature is the same as the lattice at $T_{L} \cdot\left(T_{L} \neq T_{e x}\right)$ In Sec. III $A$ we also calculate the relaxation of free $e /$ hh's to compare with that of excitons. In this case we have used monoenergetic initial momentum distributions both for $e /$ hh's and excitons. The simulation is performed in the exciton temperatures $\left(T_{e x}\right)$ ranging between 10 and $90 \mathrm{~K}$, with the lattice temperature $\left(T_{L}\right)$ and the interface roughness parameters varied. The electrons, heavy holes and excitons are treated in the parabolic band approximation as it removes the complexity of the expressions of carrier-carrier and carrier-LA phonon scattering rates, and contributes to the major reduction of computational load. Only the lowest subbands in the $\Gamma$ point of the conduction and the heavy hole band is considered in the present study, disregarding the contribution from the upper subbands and the light hole bands. Since the L-valley is located more than $280 \mathrm{meV}$ above, it does not contribute in the energy region of the present simulation. If we consider the realistic $\mathrm{QW}$ of $\mathrm{Al}_{0.3} \mathrm{Ga}_{0.7} \mathrm{As} / \mathrm{GaAs}$ with $L_{z}=10 \mathrm{~nm}$, the energy separations between the lowest hh state and the second hh state or the first light hole state is $22 \mathrm{meV}$ and $13 \mathrm{meV}$, respectively. (The separations are $33 \mathrm{meV}$ and $48 \mathrm{meV}$, respectively, for the well of the infinite barrier height.) Since the average exciton energy at the highest exciton temperature $(90 \mathrm{~K})$ is $7.7 \mathrm{meV}$, only a small fraction of excitons in the high energy tail of the Bose distribution are involved in the scatterings with the upper subbands. Thus we have expected that the upper subbands does not appreciably affect the relaxation and transport properties, and we have not included them in the simulations. The 
in-plane $\left(m_{h h / /}\right)$ and perpendicular $\left(m_{h h \perp}\right)$ (along the growth direction) effective masses in the valence band are expressed using Luttinger parameters, $\gamma_{1}$ and $\gamma_{2}, 27$

$$
m_{h h / /}=\left(\gamma_{1}+\gamma_{2}\right)^{-1} m_{0}, \quad m_{h h \perp}=\left(\gamma_{1}-2 \gamma_{2}\right)^{-1} m_{0}
$$

where $m_{0}$ is the free electron mass. The physical parameters for GaAs in the present analysis are taken from Molenkamp et al. 28 the effective mass in the conduction band $m_{e}=0.0665 m_{0}$, $\gamma_{1}=6.790$, and $\gamma_{2}=1.924$.

\section{B. Screening}

The proper treatment of the screening is essential in the many-body theory of electrons with electron-electron and electron-phonon interactions. There are various approximated

expressions with the different levels of sophistication.29.80 Among them the random phase approximation (RPA) is most commonly used since it predicts important dynamical features such as plasmons, and it has analytic expressions in 3D system both for the degenerate 29 and nondegenerate 31 carrier distributions. But the analytic expression for $2 \mathrm{D}$ system is not obtained, and we use the static expression in the present study. The original RPA expression of the dielectric function, which is valid in 2D for any distributions of carriers, is expressed as (see, e.g., Ref. 30)

$$
\varepsilon(\boldsymbol{q}, \omega)=1-V_{q} \sum_{\boldsymbol{k}, \boldsymbol{\sigma}} \frac{f_{\boldsymbol{k}-\boldsymbol{q}}-f_{\boldsymbol{k}}}{\hbar \omega+i \delta+E_{\boldsymbol{k}-\boldsymbol{q}}-E_{\boldsymbol{k}}},
$$

where $f_{\boldsymbol{k}}$ is the momentum distribution function of carriers, $E_{\boldsymbol{k}}$ is the energy of the carrier, and the summation runs over all possible states of momentum and spin. $V_{q}$ is the Fourier transform of the Coulomb potential in 2D system:

$$
V_{q}=\frac{2 \pi e^{2}}{\varepsilon_{0} L^{2} q}
$$

where $L^{2}$ is the area of normalization, and $\varepsilon_{0}$ is the dielectric constant including the contributions from the interband electronic transitions and from the phonons. In the static $(\omega \rightarrow 0)$ and the long wavelength limit $(\boldsymbol{q} \rightarrow 0)$ the RPA expression becomes, 


$$
\varepsilon(\boldsymbol{q} \rightarrow 0,0)=1-V_{q} \sum_{k, \sigma} \frac{\partial f\left(E_{q}\right)}{\partial E_{q}} .
$$

For 2D, converting the summation over $\boldsymbol{k}$ into an integral over the energy $E$ by utilizing the parabolic band dispersion, assuming an isotropic momentum distribution, and integrating by parts, Eq. (2.3) reduces to

$$
\varepsilon(\boldsymbol{q} \rightarrow 0,0)=1+\sum_{\sigma} V_{q} \frac{L^{2}}{2 \pi} \frac{2 m_{e}}{\hbar^{2}} f(E=0),
$$

which is valid for any isotropic momentum distributions of carriers. Using Eq. (2.2) and summing up spin indices, one finally obtains

$$
\varepsilon(\boldsymbol{q} \rightarrow 0,0)=1+\frac{2 m_{e} e^{2}}{\varepsilon_{0} \hbar^{2} q} f(E=0)=1+\frac{\kappa}{q},
$$

where the screening wavenumber $\kappa$ is defined by

$$
\kappa \equiv \frac{2 m_{e} e^{2}}{\varepsilon_{0} \hbar^{2}} f(E=0)
$$

This expression for the dielectric function was first adopted by Goodnick et al.32 and has been used for the EMC studies. 33 We have used this expression throughout the present study. This expression means that only the carriers in the bottom of the band contribute to the screening. The screened Coulomb interaction $V_{q}^{e f f}$ is thus described as

$$
V_{q}^{e f f} \equiv \frac{V_{q}}{\varepsilon(q \rightarrow 0,0)}=\frac{2 \pi e^{2}}{\varepsilon_{0} L^{2}}\left(\frac{1}{q+\kappa}\right)
$$

When the carriers from more than one band have to be considered, they contribute to the screening wavenumber independently in the long wavelength limit $(q \rightarrow 0)$. 3 (Cross terms appear when $q$ is finite.) Thus in the present case when the conduction and heavy hole band take part in, the screening wavenumber is expressed as

$$
\kappa=\frac{2 m_{e} e^{2}}{\varepsilon_{0} \hbar^{2}}\left[f_{e}(E=0)+f_{h h}(E=0)\right]
$$

where $f_{e}(E)$ and $f_{h h}(E)$ are the momentum distribution functions of electrons and heavy holes, respectively. The screening wavenumber is calculated by counting the number of 
electrons and heavy holes at the bottom of each band in every time step of Monte Carlo simulations. The strength of Coulomb interaction is updated every time step using this screening wavenumber.

In order to obtain the full carrier dynamics in the relaxation, the dynamic screening model is highly desirable. However, the direct calculation of Eq. (2.1) is computationally very heavy and beyond the reach of the present study. (e.g. see Bair and Krusius in Ref. 35) Instead, the static screening expression of Eq. (2.5) is used in the following simulations.

It should be noticed that the expressions for the screening is valid only in the homogeneous system. However, they can be applied to the inhomogeneous system locally when the characteristic length of the screening is much smaller than the characteristic length at which the distribution functions and the density change.36 In the present study the inverse of the screening wavenumber is $100 \mathrm{~nm}$ or less, while the characteristic length at which the exciton distribution changes is of the order of $1 \mu \mathrm{m}$. Thus we can use the locally defined screening in the present case. Furthermore, as described below, the excitons do not contribute to the screening but only the residual heavy holes, whose distribution is almost homogeneous, are responsible for the screening. Thus the effect of inhomogeneity is further reduced.

We have not included excitons in the screening in the present calculations. The screening in the presence of both charged carriers and excitons are discussed in detail by Haug and Schmitt-Rink 34 in the 3D system. In the simplest, case the dielectric function due to excitons are given by

$$
\varepsilon(\omega)=\varepsilon_{0}\left(1+4 \pi n \frac{9}{2} a_{0}^{3}\right)
$$

where $n$ and $a_{0}$ are the exciton density and Bohr radius, respectively. In the present case this yields

$$
\varepsilon=\varepsilon_{0}\left(1+5.6 \times 10^{-2}\right) .
$$

On the other hand the dielectric function due to the free carriers is

$$
\varepsilon=\varepsilon_{0}(1+O(1)) .
$$


Thus the contribution from excitons is much smaller than that from free carriers, and we have included only the free carriers in the screening.

\section{Scattering Mechanism}

\section{Carrier-Carrier Scattering}

The scattering between charged carriers is discussed in this section. The $e$-e, hh-hh, and e-hh scatterings in 2D using the Born approximation has been discussed in Ref. 19 in detail and only the results relevant to the present study are given here. The total scattering rate of an electron of a momentum $\boldsymbol{k}_{1}$ with heavy holes (momentum $\boldsymbol{k}_{2}$ ) is given by

$$
W_{e-h}\left(\boldsymbol{k}_{1}\right)=\frac{2 \pi e^{4}}{\varepsilon_{0}^{2} \hbar A} \frac{m_{r}}{\hbar^{2}} \sum_{\boldsymbol{k}_{2}, \sigma} f_{h h}\left(\boldsymbol{k}_{2}, \sigma\right) \int_{0}^{2 \pi} d \theta \frac{\left|F_{e e h h}(q)\right|^{2}}{(q+\kappa)^{2}}
$$

where $m_{r}$ is the reduced mass, $q$ is the momentum transfer from the electron to the heavy hole, and $\theta$ is the scattering angle in c.m. frame. $f_{h h}\left(\boldsymbol{k}_{2}, \sigma\right)$ is the momentum distribution function of heavy holes. The screening wavenumber $\kappa$ is given by Eq. (2.5). $\boldsymbol{q}$ is related to the initial and the final relative momentum, $\boldsymbol{k}_{r}$ and $\boldsymbol{k}_{r}^{\prime}$, and the scattering angle $\theta$ of $e$-hh system by

$$
\boldsymbol{q}=\boldsymbol{k}_{r}-\boldsymbol{k}_{r}^{\prime}, q=2 k_{r} \sin (\theta / 2)
$$

where the relative momentum is defined by

$$
\boldsymbol{k}_{r}=\frac{m_{h h / /} \boldsymbol{k}_{1}-m_{e / /} \boldsymbol{k}_{2}}{m_{h h / /}+m_{e / /}} .
$$

$F_{\text {eehh }}(q)$ is the form factor given by

$$
F_{e e h h}(q)=\int_{-\infty}^{\infty} d z_{e} \int_{-\infty}^{\infty} d z_{h}\left|\zeta_{e}\left(z_{e}\right)\right|^{2}\left|\zeta_{h}\left(z_{h}\right)\right|^{2} e^{-q\left|z_{e}-z_{h}\right|}
$$

where $\zeta_{e}\left(z_{e}\right)$ and $\zeta_{h}\left(z_{h}\right)$ are the envelope functions in the growth axis for electrons and heavy holes, respectively. The form factor is of the order of unity when only the lowest subbands of the conduction and the heavy hole bands are considered. Since carrier densities are low 
in the nondegenerate region, Pauli exclusion in the final state is not considered. The similar expression holds for $e-e$ scattering with antiparallel spins. The exchange term is included when dealing with $e$-e scattering with parallel spins. The total scattering rate in this case is given by 33

$$
W_{e-e}\left(\boldsymbol{k}_{1}\right)=\frac{2 \pi e^{4}}{\varepsilon_{0}^{2} \hbar A} \frac{m_{r}}{\hbar^{2}} \sum_{\boldsymbol{k}_{2}} f_{e}\left(\boldsymbol{k}_{2}, \sigma\right) \int_{-\frac{\pi}{2}}^{\frac{\pi}{2}} d \theta\left|\frac{F_{\text {eeee }}(q)}{q+\kappa}-\frac{F_{\text {eeee }}(Q)}{Q+\kappa}\right|^{2},
$$

where $Q$ is

$$
Q=2 k_{r} \cos (\theta / 2)
$$

The origin of the exchange effect is in the exclusion principle between the identical particles. When the two particles are identical, they cannot come close enough. Thus the short range interaction does not work between the identical particles. The scale at which the particle indistinguishability sets in can be of the order of de Broglie wavelength. In the present case, when the relative momentum between two electrons is $0.6 \mathrm{~nm}^{-1}$, the de Broglie wavelength is $10 \mathrm{~nm}$. We expect that the exchange effect becomes important when the interaction range is equal to or less than the de Broglie length. The interaction range defined by the inverse of the screening wavenumber is much longer in our case, around $100 \mathrm{~nm}$. Thus the exchange term does not affect much and the interaction strength between the electrons with parallel spin is similar to that between the electrons with antiparallel spin.

\section{Electron (Heavy Hole)-LA phonon Scattering}

The LA phonon scattering is treated in the present study since the energy of carriers and excitons is less than LO phonon energy and the lattice temperature is low $(\leq 30 \mathrm{~K})$. The $2 \mathrm{D}$ electrons interact with the phonons of bulk modes through the deformation potential. The scattering rate in $2 \mathrm{D}$ can be evaluated in parallel with the exciton-LA phonon interaction given by Takagahara in detail 37 The total LA-phonon (wavenumber $Q_{p h}$ ) absorption and emission rates from the electron with $\boldsymbol{k}$ are given by 


$$
\begin{aligned}
& W_{e-L A}^{\text {absorb }}(\boldsymbol{k})=\frac{1}{4 \pi} \frac{D_{e}^{2} m_{e / /}}{\hbar^{2} u \rho L_{z}} 2 k \int_{0}^{2 \pi} d \theta \frac{\sin \frac{\theta}{2}}{e^{\hbar u Q_{p h} / k T}-1} \\
& W_{e-L A}^{\text {emit }}(\boldsymbol{k})=\frac{1}{4 \pi} \frac{D_{e}^{2} m_{e / /}}{\hbar^{2} u \rho L_{z}} 2 k \int_{0}^{2 \pi} d \theta \sin \frac{\theta}{2}\left[\frac{1}{e^{\hbar u Q_{p h} / k T}-1}+1\right]
\end{aligned}
$$

where $u, \rho$, and $D_{e}$ are the velocity of sound, the mass density, and the deformation potential for conduction band, respectively. $\theta$ is the electron scattering angle in lab. frame. The similar expression holds for heavy hole-LA phonon scattering. Here we assume that the phonon energy $\hbar u Q_{p h}$ is much smaller than the electron kinetic energy. The z-component of the phonon momentum is always set to zero for simplicity, resulting in the slight overestimation of the scattering rate. In the present analysis the physical parameters are taken from Ref. 37; $D_{e}=-6.5 \mathrm{eV}, D_{h h}=3.1 \mathrm{eV}, \rho=5.3 \mathrm{~g} \mathrm{~cm}^{-3}, u=4.81 \times 10^{5} \mathrm{~cm} \mathrm{~s}^{-1}$.

In the present analysis the acoustic phonon distribution is always assumed to be that of thermal equilibrium at the lattice temperature, and the effects of nonequilibrium phonons emitted from the carriers are not included since the carrier density and the excitation energy

is low. There are several investigations, theoretically and experimentally, 1,38 - 41 on the effects of nonequilibrium phonons on the dynamics of carriers and excitons. This should be included in the case of high density, high excitation energy.

\section{Electron (Heavy Hole)-Interface Roughness Scattering}

The electron (heavy hole)-interface roughness (IFR) scattering in heterostructures was given by Ando et al. 2 and applied to the transport phenomena of $2 \mathrm{D}$ electron gas in QW's.43.44 Here the brief derivation is given for clarity. In the well of infinite barriers, the lowest subband energy as a function of well width is given by $E\left(L_{z}\right)=\pi^{2} \hbar^{2} /\left(2 m_{e \perp} L_{z}^{2}\right)$. When the well width changes from place to place in the 2D plane by $\Delta L_{z}(\boldsymbol{r} / /)$, the subband energy changes by

$$
\Delta E\left(\boldsymbol{r}_{/ /}\right)=\frac{\pi^{2} \hbar^{2} \Delta L_{z}\left(\boldsymbol{r}_{/ /}\right)}{m_{e \perp} L_{z}^{3}},
$$

where $\boldsymbol{r}_{/ /}$is the coordinate in $2 \mathrm{D}$ plane and $m_{e \perp}$ is the effective mass perpendicular to $2 \mathrm{D}$ plane. The fluctuation of the well width can be expanded by Fourier series as 


$$
\Delta L_{z}\left(\boldsymbol{r}_{/ /}\right)=\sum_{\boldsymbol{q}_{/ /}} \Delta_{\boldsymbol{q}_{/ /}} e^{i \boldsymbol{q}_{/ /} \boldsymbol{r}_{/ /}}
$$

$\Delta E\left(\boldsymbol{r}_{/ /}\right)$in Eq. (2.14) can be regarded as a potential for $2 \mathrm{D}$ electrons and the $e$-IFR scattering amplitude is given by its matrix element between 2D plane wave states as

$$
\left\langle\boldsymbol{k}_{/ /}^{\prime}\left|\Delta E\left(\boldsymbol{r}_{/ /}\right)\right| \boldsymbol{k}_{/ /}\right\rangle=\frac{\pi^{2} \hbar^{2}}{m_{e \perp} L_{z}^{3}} \Delta_{\boldsymbol{q}_{/ /}} .
$$

where $\boldsymbol{k}_{/ /}-\boldsymbol{k}_{/ /}^{\prime}=\boldsymbol{q}_{/ /}$. The Gaussian correlation function of the well-width fluctuation is assumed, thus

$$
\left\langle\Delta L_{z}\left(\boldsymbol{r}_{/ /}\right) \cdot \Delta L_{z}\left(\boldsymbol{r}_{/ /}^{\prime}\right)\right\rangle=\Delta^{2} \exp \left[\left(\boldsymbol{r}_{/ /}-\boldsymbol{r}_{/ /}^{\prime}\right)^{2} / \Lambda^{2}\right]
$$

where $\Delta$ and $\Lambda$ are the amplitude and the correlation length of the fluctuation, respectively. Using the Fourier expansion Eq. (2.15), the left side reduces to

$$
\left\langle\sum_{\boldsymbol{q}_{/ /}, \boldsymbol{q}_{/ /}^{\prime}} \Delta_{\boldsymbol{q}_{/ /}} \Delta_{\boldsymbol{q}_{/ /}^{\prime}} e^{i\left(\boldsymbol{q}_{/ /} \cdot \boldsymbol{r}_{/ /}+\boldsymbol{q}_{/ /}^{\prime}, \boldsymbol{r}_{/ /}^{\prime}\right)}\right\rangle=\sum_{\boldsymbol{q}_{/ /}}\left|\Delta_{\boldsymbol{q}_{/ /}}\right|^{2} e^{i \boldsymbol{q}_{/ /} \cdot \Delta \boldsymbol{r}_{/ /}}
$$

The right side can be expanded in Fourier series, then Eq. (2.17) reduces to

$$
\sum_{\boldsymbol{q}_{/ /}}\left|\Delta \boldsymbol{q}_{/ /}\right|^{2} e^{i \boldsymbol{q}_{/ /} \cdot \Delta \boldsymbol{r}_{/ /}}=\sum_{\boldsymbol{q}_{/ /}} \frac{1}{A} \pi \Lambda^{2} \Delta^{2} e^{-q_{/ /}^{2} \Lambda^{2} 4} e^{i \boldsymbol{q}_{/ /} \cdot \Delta \boldsymbol{r}_{/ /}}
$$

Thus the expansion coefficient is expressed as

$$
\left|\Delta \boldsymbol{q}_{/ /}\right|^{2}=\frac{1}{A} \pi \Lambda^{2} \Delta^{2} e^{-q_{/ /}^{2} \Lambda^{2} / 4}
$$

Using this in the right side of Eq. (2.16), the square of matrix element is written as

$$
\left|\left\langle\boldsymbol{k}_{/ /}^{\prime}\left|\Delta E\left(\boldsymbol{r}_{/ /}\right)\right| \boldsymbol{k}_{/ /}\right\rangle\right|^{2}=\frac{\pi^{5} \hbar^{4} \Lambda^{2} \Delta^{2}}{A m_{e \perp}^{2} L_{z}^{6}} e^{-q_{/ /}^{2} \Lambda^{2} / 4}
$$

The total scattering rate of the electron with momentum $\boldsymbol{k}$ is calculated via Fermi's golden rule as

$$
W_{e-I F R}\left(\boldsymbol{k}_{/ /}\right)=\frac{\pi^{4} \hbar \Lambda^{2} \Delta^{2} m_{e / /}}{2 m_{e \perp}^{2} L_{z}^{6}} \int_{0}^{2 \pi} d \theta e^{-k_{/ /}^{2} \Lambda^{2} \sin ^{2}(\theta / 2)}
$$

where $\theta$ is the scattering angle in Lab. frame. The experimental determination of IFR parameters, $\Delta$ and $\Lambda$, is very difficult. The former is the well width fluctuation and usually 
one or two monolayers is assumed. The latter corresponds to the typical terrace or island size in the two dimensional plane. In the present simulations $\Delta$ is $0.283 \mathrm{~nm}$ (one monolayer) and $\Lambda$ is $10 \mathrm{~nm}$ unless otherwise stated. In this model, since the infinite barrier height is assumed, the IFR scattering is somewhat overestimated compared with the realistic well of a finite barrier height.

\section{Exciton-Electron (Heavy Hole) Scattering}

We derive here the exciton-electron (ex-e) scattering to the lowest order. The exciton wavefunction in the ideal 2D system (z-dependence ignored) is written as

$$
|\boldsymbol{K}\rangle=\frac{1}{\sqrt{A}} \sum_{\boldsymbol{r}_{e}, \boldsymbol{r}_{h}} e^{i \boldsymbol{K} \cdot \boldsymbol{R}_{F}}\left(\boldsymbol{r}_{e}, \boldsymbol{r}_{h}\right) c_{\boldsymbol{r}_{e}}^{\dagger} b_{\boldsymbol{r}_{h}}^{\dagger}|0\rangle,
$$

where $\boldsymbol{K}$ and $\boldsymbol{R}$ are the center-of-mass momentum and coordinate, respectively. $F$ is the wavefunction of relative motion, and $c_{\boldsymbol{r}_{e}}^{\dagger}\left(b_{\boldsymbol{r}_{h}}^{\dagger}\right)$ is the electron (heavy hole) creation operator in the Wannier representation. The wavefunction can be rewritten in the Bloch representation using the relation

$$
c_{\boldsymbol{r}_{e}}^{\dagger}=\frac{1}{\sqrt{N}} \sum_{\boldsymbol{k}} e^{-i \boldsymbol{k} \cdot \boldsymbol{r}_{e}} c_{\boldsymbol{k}}^{\dagger},
$$

where $N$ is the number of unit cells in the area $A, A=N v_{0}\left(v_{0}\right.$ is the area of the unit cell). Converting the summation over lattice sites into the integral by

$$
\sum_{\boldsymbol{r}} \rightarrow \frac{1}{v_{0}} \int d^{2} \boldsymbol{r}
$$

the exciton wavefunction reduces to

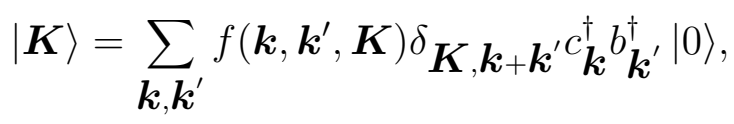

where

$$
f\left(\boldsymbol{k}, \boldsymbol{k}^{\prime}, \boldsymbol{K}\right)=\frac{N}{A^{3 / 2}} \int d^{2} \boldsymbol{r} F(\boldsymbol{r}) e^{i\left(\alpha_{e} \boldsymbol{K}-\boldsymbol{k}\right) \cdot \boldsymbol{r}}
$$

The mass ratios $\alpha_{e}$ and $\alpha_{h}$ are defined by

$$
\alpha_{e}=\frac{m_{e}}{m_{e}+m_{h}}, \quad \alpha_{h}=\frac{m_{h}}{m_{e}+m_{h}} .
$$


We evaluate ex-e (or ex-hh) scattering to the lowest order, assuming that there happens neither rearrangement of electrons nor the excitations of the internal state of the exciton, and ignoring exchange effect. The Coulomb interaction Hamiltonian is written as (spin indices are dropped)

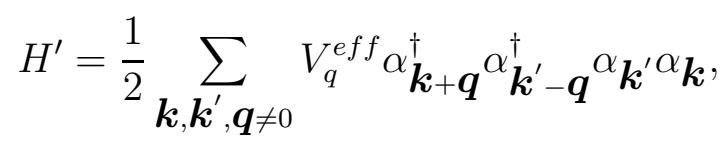

where $\alpha_{\boldsymbol{k}}^{\dagger}$ is an operator for electrons or heavy holes. $V_{q}^{\text {eff }}$ is the screened Coulomb potential of Eq. (2.7), where the screening in only due to the free $e / \mathrm{hh}$ 's. The scattering amplitude is obtained by calculating the matrix element of $H^{\prime}$ between the initial and the final electronexciton states, $|\boldsymbol{k}, \boldsymbol{K}\rangle$ and $\left|\boldsymbol{k}^{\prime}, \boldsymbol{K}^{\prime}\right\rangle$. Here $\boldsymbol{k}\left(\boldsymbol{k}^{\prime}\right)$ and $\boldsymbol{K}\left(\boldsymbol{K}^{\prime}\right)$ represent the initial (final) electron and exciton momentum, respectively. Two terms remain and the corresponding diagrams are shown in (a) and (b) of Fig. 1. The term described by (a) is

$$
\sum_{\boldsymbol{k}_{1}, \boldsymbol{k}_{2}, \boldsymbol{l}_{1}, \boldsymbol{l}_{2}} V_{q} f^{*}\left(\boldsymbol{l}_{1}, \boldsymbol{l}_{2}, \boldsymbol{K}^{\prime}\right) f\left(\boldsymbol{k}_{1}, \boldsymbol{k}_{2}, \boldsymbol{K}\right) \delta_{\boldsymbol{K}^{\prime}, \boldsymbol{l}_{1}+\boldsymbol{l}_{2}}{ }^{\delta} \boldsymbol{K}, \boldsymbol{k}_{1}+\boldsymbol{k}_{2}{ }^{\delta} \boldsymbol{q}, \boldsymbol{l}_{1}-\boldsymbol{k}_{1} \delta_{\boldsymbol{l}_{2}, \boldsymbol{k}_{2}} .
$$

This term can be evaluated by using Eq. (2.21) and converting the summation over momenta into the integral. Evaluating two terms (a) and (b), the matrix element is give by

$$
\left\langle\boldsymbol{k}_{3}-\boldsymbol{q}, \boldsymbol{K}+\boldsymbol{q}\left|H^{\prime}\right| \boldsymbol{k}_{3}, \boldsymbol{K}\right\rangle=\frac{V_{q} N^{2}}{A^{2}} \int d^{2} \boldsymbol{r}|F(r)|^{2}\left[e^{i \alpha_{h} \boldsymbol{q} \cdot \boldsymbol{r}}-e^{-i \alpha_{e} \boldsymbol{q} \cdot \boldsymbol{r}}\right] .
$$

Using the internal wavefunction of the lowest energy, s-wave exciton; $F(r)=2 \alpha v_{0} e^{-\alpha r} / \sqrt{2 \pi}$, where $\alpha$ is the inverse of the Bohr radius, Eq. (2.22) reduces to

$$
\frac{V_{q} N^{2}}{A^{2}} \frac{4 \alpha^{2} v_{0}^{2}}{2 \pi} \int r d r d \theta e^{-2 \alpha r}\left[e^{i \alpha_{h} \boldsymbol{q} \cdot \boldsymbol{r}}-e^{-i \alpha_{e} \boldsymbol{q} \cdot \boldsymbol{r}}\right] .
$$

This expression can be integrated by using the generation function of the Bessel function, and one finally obtains for the matrix element

$$
\left\langle\boldsymbol{k}_{3}-\boldsymbol{q}, \boldsymbol{K}+\boldsymbol{q}\left|H^{\prime}\right| \boldsymbol{k}_{3}, \boldsymbol{K}\right\rangle=V_{q}\left\{\frac{1}{\left[1+\left(\frac{\alpha_{h} q}{2 \alpha}\right)^{2}\right]^{3 / 2}}-\frac{1}{\left[1+\left(\frac{\alpha_{e} q}{2 \alpha}\right)^{2}\right]^{3 / 2}}\right\} .
$$

The total scattering rate of an exciton with electrons (heavy holes) can be calculated with the Fermi's golden rule as 


$$
W_{e x-e, h}(\boldsymbol{K})=\frac{2 \pi e^{4} m_{r}}{\hbar^{3} \varepsilon_{0}^{2} A} \sum_{\boldsymbol{k}} f_{e, h}(\boldsymbol{k}) \int_{0}^{2 \pi} d \theta \frac{1}{(q+\kappa)^{2}}\left\{\frac{1}{\left[1+\left(\frac{\alpha_{h} q}{2 \alpha}\right)^{2}\right]^{3 / 2}}-\frac{1}{\left[1+\left(\frac{\alpha_{e} q}{2 \alpha}\right)^{2}\right]^{3 / 2}}\right\}^{2}
$$

where $m_{r}$ is the reduced mass of an exciton and electron (heavy hole), $f_{e, h}(\boldsymbol{k})$ is the momentum distribution function of electrons (heavy holes), and $\kappa$ is the screening wavenumber defined in Eq. (2.6). The scattering angle $\theta$ is related to the momentum transfer $q$ and the relative momentum $m_{r}$ as $q=2 k_{r} / \sin (\theta / 2)$. The exciton Bohr radius $\alpha^{-1}$ is estimated to be $12.5 \mathrm{~nm}$ from the variational calculation. 45

\section{Exciton-Exciton Scattering}

We can derive the exciton-exciton (ex-ex) scattering in the similar manner as ex-e scattering described in the previous section. The relevant diagrams are shown in (c)-(f) of Fig. 1. The total scattering rate is given as

$W_{e x-e x}\left(\boldsymbol{K}_{\alpha}\right)=16 \frac{2 \pi e^{4} m_{r}}{\hbar^{3} \varepsilon_{0}^{2} A} \sum_{\boldsymbol{K}_{\beta}} f_{e x}\left(\boldsymbol{K}_{\beta}\right) \int_{0}^{2 \pi} d \theta \frac{1}{(q+\kappa)^{2}}\left\{\frac{1}{\left[1+\left(\frac{\alpha_{h} q}{2 \alpha}\right)^{2}\right]^{3 / 2}}-\frac{1}{\left[1+\left(\frac{\alpha_{e} q}{2 \alpha}\right)^{2}\right]^{3 / 2}}\right\}^{4}$,

where $\boldsymbol{K}_{\alpha}$ is the momentum of the exciton, $m_{r}$ is the reduced mass of two excitons, and $f_{\text {ex }}(K)$ is the momentum distribution function of excitons.

Comparing the exciton scattering rates, Eq. (2.25) and Eq. (2.26), with the electron scattering rate, Eq. (2.9), they show large differences in their magnitude and $q$-dependence. Figure 2 shows the plots of the scattering rates of the three processes vs. $q$. The $e$-hh scattering rate is forward peaked, and is larger than other two processes by several orders of magnitude. The magnitudes of ex-hh and ex-ex scattering rise with $q$ and reach the peak at $q=0.15$ and $0.20 \mathrm{~nm}^{-1}$, respectively, and are much smaller than $e$-hh scattering. These features of scattering mechanism lead to the striking difference in the relaxation and transport between electrons and excitons as described later. 


\section{Exciton-LA Phonon Scattering}

There is a detailed theoretical study of Takagahara 37 on the exciton-LA phonon interaction and only the results relevant to the present calculations are shown here. The total exciton scattering rates for the phonon absorption and emission processes have the similar forms as the electron case as,

$$
\begin{aligned}
& W_{\text {ex-LA }}^{\text {absorb }}(\boldsymbol{k})=\frac{1}{4 \pi} \frac{\left(D_{e}-D_{h h}\right)^{2} m_{e / /}}{\hbar^{2} u \rho L_{z}} 2 k \int_{0}^{2 \pi} d \theta \frac{\sin \frac{\theta}{2}}{e^{\hbar u Q_{p h} / k T}-1} \\
& W_{\text {exit }-L A}^{\text {emit }}(\boldsymbol{k})=\frac{1}{4 \pi} \frac{\left(D_{e}-D_{h h}\right)^{2} m_{e / /}}{\hbar^{2} u \rho L_{z}} 2 k \int_{0}^{2 \pi} d \theta \sin \frac{\theta}{2}\left[\frac{1}{e^{\hbar u Q_{p h} / k T}-1}+1\right]
\end{aligned}
$$

where the notations are the same as electron-LA phonon scattering.

\section{Exciton-Interface Roughness Scattering}

The exciton-IFR scattering is deduced from the electron-IFR scattering. Using the matrix element of Eq. (2.18), the electron-IFR interaction Hamiltonian can be written as

$$
H_{e x-I F R}^{\prime}=\sum_{\boldsymbol{k}_{/ /}, \boldsymbol{q}_{/ /}} \frac{\pi^{5 / 2} \hbar^{2} \Lambda \Delta}{A^{1 / 2} L_{z}^{3}} e^{-q_{/ /}^{2} \Lambda^{2} / 2}\left(\frac{1}{m_{e \perp}} c_{\boldsymbol{k}_{/ /}^{\dagger}+\boldsymbol{q}_{/ /}}{ }^{c} \boldsymbol{k}_{/ /}+\frac{1}{m_{h h \perp}} b_{\boldsymbol{k}_{/ /+}^{\dagger}}^{\dagger} \boldsymbol{q}_{/ /}{ }^{b} \boldsymbol{k}_{/ /}\right)
$$

where $c^{\dagger}$ and $b^{\dagger}$ are operators for an electron and a heavy hole, respectively. The derivation is similar to the case of the $e$-IFR scattering and the total scattering rate is given by

$$
W_{e x-I F R}(\boldsymbol{K})=\frac{\pi^{4} \hbar \Delta^{2} \Lambda^{2} m_{e x / /}}{2 L_{z}^{6}}\left(\frac{1}{m_{e \perp}}-\frac{1}{m_{h h \perp}}\right)^{2} \int_{0}^{2 \pi} d \theta e^{-K_{/ /}^{2} \Lambda^{2} \sin ^{2} \frac{\theta}{2}} .
$$

\section{Simulation Method}

The EMC simulations in $k$-space have been widely used in the analysis of photogenerated carriers 1724 and reviewed in Ref. 15, and the detailed technique is not repeated here. In order to trace the distributions of the particles in $r$-space within the frame work of the $k$-space EMC method, the simulation area is divided into ten concentric circular regions as shown in Fig. 3. In each region the average particle density and the momentum distribution 
function are calculated in every time step and the $k$-space EMC simulation is performed, independently from other regions, with the scattering parameters corresponding to the average density. (Interparticle scatterings are affected directly through their dependence on the density and indirectly through the change in the screening wavenumber. Other scatterings are also affected indirectly through the change of the momentum distribution of particles.) The positions, as well as the momenta, of particles are updated after each time step. A particle can move to the neighboring region, thus the particle density, and consequently the scattering parameters, change every time step. This technique allows one to trace the time evolution of both the spatial and the momentum distribution of particles with relatively low computational load. The disadvantage is that, since the continuous density distribution (solid line in Fig. 3) is approximated by the step-like one, the difference of the scattering rates between the particles near the inner boundary and the outer boundary of the region is neglected. Thus the spatial distribution of particles calculated in the present method is only valid in the length scale larger than the region size. The range of the Coulomb interaction,

determined by the inverse of the screening wavenumber is of the order of $100 \mathrm{~nm}$ in the present simulations, which is much smaller than the region size.

In the present study ten or twenty simulation areas as mentioned above are prepared and simulated simultaneously. The physical information is obtained by taking their ensemble average. The number of particles is 78600 when twenty simulation areas are used. The time step of simulations are 5 fs for free $e /$ hh's and 12.5 fs for excitons. We have confirmed that the time step is much smaller than the scattering interval and that the simulation results do not change by further reducing the time step.

\section{RESULTS AND DISCUSSION}




\section{A. Electron and Exciton Relaxations}

It is expected that the scattering frequency and thus the energy relaxation is different between free $e /$ hh's and excitons reflecting the different magnitude of scattering processes. To see this, the frequency of each scattering process is counted from 0 to 15 ps both for electrons and excitons. For $e /$ hh's the simulation is done in the mono-energetic initial momentum distribution, with the excitation energy $10 \mathrm{meV}$ above the band gap (the excess energies is $6.34 \mathrm{meV}$ for electrons and $3.66 \mathrm{meV}$ for heavy holes) in the presence of residual hh's at the lattice temperature $5 \mathrm{~K}$. The spatial distribution is in the Gaussian function with FWHM $6 \mu \mathrm{m}$ as with excitons. The similar simulation is performed for excitons assuming that they were generated mono-energetically at $10 \mathrm{meV}$. Notice that this is physically not realistic because it is not possible to optically generate excitons except at the bottom of the band due to the energy-momentum conservation. It is calculated to show the remarkable difference between the electron and exciton relaxation due to the difference in the magnitude of interparticle scatterings.

Plotted in Fig. 1 are the numbers of each scattering events per particle per second. For electrons $e$-hh, $e$-e and $e$-IFR scatterings are of the similar magnitude of $1 \times 10^{12} \mathrm{~s}^{-1}$. (The frequency the $e$-LA phonon scatterings is several orders of magnitude smaller than those plotted here.) The $e$-hh scattering frequency increases in the initial few ps reflecting the rapid energy relaxation of electrons: When the electrons have larger momenta and there are many residual hh's with very small average momentum just after photogeneration, $e$-hh scattering is less frequent because it favors the small momentum transfer.

The exciton scatterings plotted in the lower part of Fig. Đ show different features. (Notice that they are plotted in logarithmic scale.) While the ex-IFR scattering is as frequent as $e$-IFR scattering, the interparticle scatterings as ex-ex and ex-hh scatterings are more than two orders of magnitude smaller than those of electrons. This is due to the small scattering rates of excitons as shown in Fig. 2. Thus the contributions from the interparticle scatterings (and LA phonon scatterings which is not shown in Fig. Th the figure becomes too busy) is 
very small. The dominance of IFR scattering in excitons is in agreement with the analytic calculations of Basu et al.14

The difference of the interparticle scattering rates in free $e / \mathrm{hh}$ and exciton system has significant effect on the time evolutions of the momentum distribution functions. Figure 5 shows the distribution functions of the initial 15 ps. The electrons, initially generated at $0.105 \mathrm{~nm}^{-1}(=6.337 \mathrm{meV})$, relax rapidly, and approach the thermal distribution described by the Boltzmann distribution function (with the electron temperature $T_{e}$ ) at 15 ps. This is due to the high rate of $e$-hh and $e$-e scatterings which are quite efficient in redistributing kinetic energies among electrons and heavy holes. It should be noticed that both the electrons and the heavy holes are in equilibrium, with the same temperature $T_{e}$. (The thermalization with the lattice is achieved through the interaction with LA-phonons. But this process is quite slow, more than two orders of magnitude lower than $e$-e scattering. Thus the electron temperature is larger than the lattice temperature at this stage.) On the other hand the exciton distribution does not change appreciably in the time scale shown here. The ex-IFR scattering, which is the dominant process for excitons, is an elastic scattering, and does not change the kinetic energy (or the magnitude of momentum) of excitons. Thus the energy relaxation is induced only through very slow ex-hh and LA phonon scatterings. Another feature attributed to the difference of the scattering processes is that the electron transport would depend on the density while the exciton transport would not.

\section{B. Exciton Transport for 500 ps}

The spatial transport and the energy relaxation of excitons have been calculated up to 500 ps. Here the initial excitons are in the Bose distribution with the temperature $T_{e x}$ while the initial residual heavy holes are in the Fermi distribution at $T_{L}$ in equilibrium with the lattice. Figure 6 shows the time evolution in the momentum distribution functions of excitons up to $500 \mathrm{ps}$ with $25 \mathrm{ps}$ step, at $T_{e x}=30 \mathrm{~K}$ and the lattice temperature is $5 \mathrm{~K}$. Because of the low scattering rates of ex-ex, ex-hh, and ex-LA phonon processes, the energy 
relaxation is slow, and the excitons do not reach the thermal equilibrium with the lattice even at 500 ps. Thus the analytic calculations of the exciton transport which assume the momentum distribution in equilibrium with the lattice can underestimate the diffusivity in the earlier stage.

We have obtained the full width at half maximum (FWHM) of the exciton spatial distribution by fitting the Gauss function to the distribution calculated from the simulations. Figure 7 shows the time evolution of the spatial spread (FWHM) of excitons at several initial exciton temperatures $\left(T_{e x}=10 \mathrm{~K}-90 \mathrm{~K}\right)$ from 0 ps to 500 ps when the initial residual hh's (and the lattice) are at $5 \mathrm{~K}$. The fitting uncertainty in FWHM is around $0.03 \mu \mathrm{m}$. The calculations show that the excitons spread faster when the initial exciton temperature is higher. This trend can be easily understood when we remember that the dominant scattering process for excitons is the ex-IFR scattering. The ex-IFR scattering probability (Eq. (2.30) ) does not depend on the exciton density and is weakly dependent on the exciton momentum. Thus the the mean free path of excitons due to the ex-IFR scattering is similar at any place in the simulation area and does not vary significantly during the simulation from 0 to 500 ps. In this case, the transport is essentially determined by the velocities of excitons, with which they travel during consecutive IFR scatterings. Since the average velocity is directly related to the exciton temperature, the transport becomes enhanced with the temperatures. This also means that, in the short time scale where the exciton temperature is virtually constant, the transport can be regarded as diffusive motion characterized by the constant diffusion coefficient, $D=\langle v\rangle \lambda / 2$, over whole simulation area. Here $\langle v\rangle$ is an average velocity and $\lambda$ is a mean free path. If excitons were to go through diffusive motion with a constant diffusion coefficient and the initial spatial distribution were Gaussian, they would spread with time $t$ retaining Gaussian-shaped distribution with its $F W H M(t)$ given by $\sqrt{16 \ln 2 \times D t+F W H M(0)^{2}}$. We have also plotted this curve with the diffusion constant $30 \mathrm{~cm}^{2} \mathrm{~s}^{-1}$ in Fig. 7 with a broken line for reference. The FWHM with a constant diffusion coefficient is almost linear in the scale of Fig. 7, in contrast to the sublinear trend of the simulation results. This can be interpreted that the diffusion coefficient decreases 
dynamically due to the reduction of the average velocity. This is because the initial exciton temperatures are higher than the initial temperature of residual hh's or the lattice, the exciton temperature (and their average velocity) decreases through the ex-hh and ex-LA phonon interactions. The diffusion coefficient of the initial stage $(0-25 \mathrm{ps})$ is $180 \mathrm{~cm}^{2} \mathrm{~s}^{-1}$ for $T_{e x}=90 \mathrm{~K}$, while it reduces to $5 \mathrm{~cm}^{2} \mathrm{~s}^{-1}$ at $475-500 \mathrm{ps}$.

In Fig. 8 we have plotted the spatial spread of excitons when $T_{L}$ (the initial residual hh temperature and the lattice temperature) is varied. In the upper plot, when $T_{e x}(30 \mathrm{~K})$ is larger than $T_{L}(5 \mathrm{~K})$ (solid curve), the FWHM is sublinear, reflecting the cooling down of excitons by the residual hh's and the lattice. When $T_{e x}=T_{L}=30 \mathrm{~K}$ (broken curve), the FWHM increases linearly because there is no net energy flow among excitons, residual hh's and the lattice. Actually in this case the FWHM evolution can be reproduced by the simple diffusion model with a constant diffusivity $D=19 \mathrm{~cm}^{2} \mathrm{~s}^{-1}$. On the other hand, in the lower plot, when the lattice temperature $(30 \mathrm{~K})$ is higher than the initial exciton temperature $(10$ K) (broken curve), the FWHM evolution is superlinear because the excitons are heated by the residual hh's and the lattice.

In the calculations above, the IFR parameters were fixed $(\Delta=0.283 \mathrm{~nm}, \Lambda=10 \mathrm{~nm})$. We have done further calculations to investigate how the interface roughness affects the transport phenomena. In the present model the interface roughness is characterized by the two parameters; $\Lambda$ and $\Delta$. The former designates the typical lateral size of terrace or island in the 2D plane of QW's, while the latter indicates the terrace height. There are several experimental investigations to observe the interface structures in QW's 4 世, but it is still a controversial problem. We have used several typical values of the parameters in the simulations. Figure 9 shows the FWHM of excitons at $T_{e x}=90 \mathrm{~K}$ and $T_{L}=5 \mathrm{~K}$. In the upper plot the calculations with $\Lambda=5,10$, and $20 \mathrm{~nm}$ are compared. ( $\Delta$ is fixed at one monolayer, $0.283 \mathrm{~nm}$ ) The diffusion coefficient obtained by fitting to the simple diffusion model at the initial stage $(0-25 \mathrm{ps})$ is 100,170 , and $250 \mathrm{~cm}^{2} \mathrm{~s}^{-1}$ for $\Lambda=5$, 10, and 20 $\mathrm{nm}$, respectively. The lower plot shows the $\Delta$ dependence (one monolayer; $0.283 \mathrm{~nm}$, and two-monolayer; $0.566 \mathrm{~nm}$ ) of the transport when $\Lambda$ is fixed at $10 \mathrm{~nm}$. The initial diffusion 
coefficient is 170 an $60 \mathrm{~cm}^{2} \mathrm{~s}^{-1}$, respectively. These results show that the transport strongly depends on the IFR parameters (the spatial spread is faster when the lateral terrace size is larger and the terrace height is lower), and thus the transport properties would vary from sample to sample depending on the growth conditions.

We shall discuss the limitations of the present model with regard to the realistic situation in the experiment. The experimentally observed diffusion coefficients vary widely from few $\mathrm{cm}^{2} \mathrm{~s}^{-1}$ to several hundreds of $\mathrm{cm}^{2} \mathrm{~s}^{-1}$ depending on the experimental conditions as sample temperatures, excitation energies, carrier densities, and the sample structures. direct comparison of the present simulations with the experimental observation is difficult because, in experiments, free electrons and heavy holes are initially photogenerated, then they form bound system, excitons. Thus we should have started simulations from free $e / h$ h's and handled the exciton formation process properly. However, though there are several experimental studies on the exciton formation time 49 52, to the best of our knowledge, the theoretical formation model is not available. Here we can compare the simulation results with experiments with the similar carrier density conditions only in qualitative manner, assuming that if the initial excitation energy is higher, the resultant exciton temperature is also high. In Ref. 0,11, the experimental diffusivity decreases with the excitation energy. The trend

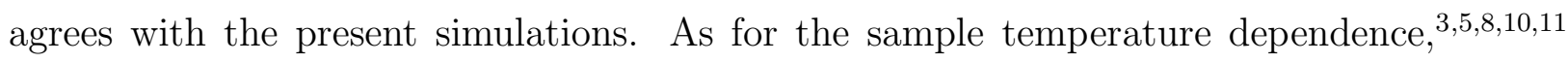
the experimental diffusivity decreases with sample temperatures down to $20 \mathrm{~K}$, which also agrees with the simulation results if we assume that the exciton temperature becomes lower with the sample temperatures. However, below $20 \mathrm{~K}$, Ref. 10,11 report the increase of the diffusivity, which is not observed in the other experiments. Our simulation results does not reproduce the diffusivity enhancement below $20 \mathrm{~K}$. The origin of the enhancement is not understood yet.

For the case of very lower exciton energy, we have to consider the validity of the present IFR scattering model. In the ex-IFR scattering an exciton is treated in a plane wave mode, extending over all 2D plane. However it is experimentally observed 16 过 that excitons become localized in the potential minima of the interface roughness in 2D plane with the reduction 
of the in-plane kinetic energy. The localized excitons can still migrate in the 2D plane by phonon-assisted hopping or variable range hopping (in the very low temperatures). This causes the significant slowing down of the diffusivity 5 . The present model does not include this effect. This becomes important when the exciton temperature gets sufficiently low by emitting phonons.

\section{CONCLUSIONS}

The time dependence of the spatial distributions of nonequilibrium excitons was obtained using the ensemble Monte Carlo simulations including the interparticle scatterings (ex-ex, ex-carrier carrier-carrier), LA phonon emissions/absorptions, and the IFR scatterings. The simulations show that the dominant scattering process for excitons is the ex-IFR scattering, in contrast to charged carriers for which the carrier-carrier scattering is most significant. The difference of the dominant scattering processes affects the energy relaxations: The $e /$ hh system approaches the quasi-thermal equilibrium among carriers very rapidly (in few picoseconds) through the efficient energy redistribution caused by the carrier-carrier collisions. In contrast, the excitons relax very slowly because their main scattering process, the ex-IFR scattering, is elastic and does not change exciton energies. Excitons can exchange energies only through the ex-ex, ex-hh and ex-LA phonon scatterings with very low probabilities.

The transport of excitons is essentially determined by their average velocity because the IFR scattering is weakly dependent on the exciton distributions and the effect of other scattering processes is negligibly small. Thus the exciton transport can be regarded a diffusive motion whose diffusion coefficient varies with time. The diffusion coefficient, which is proportional to the average velocity, varies through the energy exchange with residual hh's and lattice. The spatial transport is strongly dependent on the IFR parameters; the lateral terrace size $\Lambda$ and the terrace height $\Delta$. The transport is quenched when the terrace size is smaller or the terrace height is higher. This means that the exciton transport properties are sensitive to the interface structures, and thus, to the growth conditions of QW's. 


\section{ACKNOWLEDGMENTS}

We acknowledge Koji Muraki for valuable discussions. We also acknowledge Makoto Takahashi for his offer of the computer resources. This work was partly supported by the Grant-in-aid for Scientific Research No. 07855002 from the Ministry of Education, Science and Culture, Japan. 


\section{REFERENCES}

* E-mail Address: takahasy@eie.yz.yamagata-u.ac.jp

${ }^{1}$ H.F. Hess, E. Betzig, T. D. Harris, L. N. Pfeiffer, and K. W. West, Science 264, 1740 (1994).

${ }^{2}$ J. Hegarty, L. Goldner, and M. D. Sturge, Phys. Rev. B 30, 7346 (1984).

${ }^{3}$ D. Oberhauser, K.-H. Pantke, J. M. Mvam, G. Weimann, and C. Klingshirn, Phys. Rev. B 47, 6827 (1993).

${ }^{4}$ L. M. Smith, J. S. Preston, J. P. Wolfe, D. R. Wake, J. Klem, T. Henderson, and H. Morkoç, Phys. Rev. B 39, 1862 (1989).

${ }^{5}$ H. W. Yoon, D. R. Wake, J. P. Wolfe, and H. Morkoç, Phys. Rev. B 46, 13461 (1992).

${ }^{6}$ K. T. Tsen, O. F. Sankey, G. Halama, Shu-Chen Y. Tsen, and H. Morkoç, Phys. Rev. B 39, 6276 (1989).

${ }^{7}$ K. T. Tsen, Mod. Phys. Lett. B 6, 703 (1992).

${ }^{8}$ H. Hillmer, A. Forchel, S. Hansmann, M. Morohashi, E. Lopez, H. P. Meier, and K. Ploog, Phys. Rev. B 39, 10901 (1989).

${ }^{9}$ H. Hillmer, A. Forchel, R. Sauer, and C. W. Tu, Phys. Rev. B 42, 3220 (1990).

${ }^{10}$ H. Hillmer, A. Forchel, and C. W. Tu, Phys. Rev. B 45, 1240 (1992).

${ }^{11}$ Yutaka Takahashi, Koji Muraki, Susumu Fukatsu, Satoru Kano, Yasuhiro Shiraki, and Ryoichi Ito, Solid State Commun. 88, 677 (1993).

${ }^{12}$ Yutaka Takahashi, Koji Muraki, Susumu Fukatsu, Satoru Kano, Yasuhiro Shiraki, and Ryoichi Ito, Jpn. J. Appl. Phys. 32, 5586 (1993).

${ }^{13}$ Hidefumi Akiyama, Toshio Matsusue, and Hiroyuki Sakaki, Phys. Rev. B 49, 14523 (1994).

${ }^{14}$ P. K. Basu and Partha Ray, Phys. Rev. B 44, 1844 (1991). 
15 J. Shah, in Hot Carriers in Semiconductor Nanostructures, edited by J. Shah, (Academic Press, 1992), and references therein.

${ }^{16}$ Carlo Jacoboni and Lino Reggiani, Rev. Mod. Phys. 55, 645 (1983).

${ }^{17}$ P. Lugli and S. M. Goodnick, Phys. Rev. Lett. 59, 716 (1987).

${ }^{18}$ P. Lugli, C. Jacoboni, and P. Kocevar, Appl. Phys. Lett. 50, 1251 (1987).

19 S. M. Goodnick and P. Lugli, Phys. Rev. B 37, 2578 (1988).

${ }^{20}$ M. Artaki and K. Hess, Phys. Rev. B 37, 2933 (1988).

${ }^{21}$ S. M. Goodnick and P. Lugli, Phys. Rev. B 38, 10135 (1988). The carrier-carrier interaction is overestimated by a factor of four in this reference. We have used the corrected one in the present simulations.

${ }^{22}$ P. Lugli, P. Bordone, L. Reggiani, M. Rieger, P. Kocevar, and S. M. Goodnick, Phys. Rev. B 39, 7852 (1989).

${ }^{23}$ D. W. Bailey, C. J. Stanton, and K. Hess, Phys. Rev. B 42, 3423 (1990).

24 Tilmann Kuhn and Fausto Rossi, Phys. Rev. B 46, 3423 (1992).

${ }^{25}$ Lucio Rota, Paolo Lugli, Thomas Elsaesser, and Jagdeep Shah, Phys. Rev. B 47, 4226 (1993).

${ }^{26}$ M. G. Kane, K. W. Sun, and S. A. Lyon, Phys. Rev. B 50, 7428 (1994).

27 J. M. Luttinger, Phys. Rev. 102, 1030 (1956).

${ }^{28}$ L. W. Molenkamp, R. Eppenga, G. W. 't Hooft, P. Dawson, C. T. Foxon, and K. J. Moore, Phys. Rev. B 38, 4314 (1988).

${ }^{29}$ G. D. Mahan, Many-Particle Physics (Plenum, New York, 1990).

${ }^{30} \mathrm{H}$. Haug and S. W. Koch, Quantum Theory of the Optical and Electronic Properties of 
Semiconductors (World Scientific, Singapore, 1990).

${ }^{31}$ J. R. Meyer and F. J. Bartoli, Phys. Rev. B 28, 915 (1983).

${ }^{32}$ S. M. Goodnick and P. Lugli, Appl. Phys. Lett. 51, 584 (1987).

${ }^{33}$ M. Moško and A. Mošková, and V. Cambel, Phys. Rev. B 51, 16860 (1995).

${ }^{34}$ H. Haug and S. Schmitt-Rink, Prog. Quant. Electr. 9, 3 (1984).

${ }^{35}$ J. E. Bair and J. P. Krusius, Proc. SPIE 1677, 157 (1992).

${ }^{36}$ R. W. Hockney and J. W. Eastwood, Computer Simulation Using Particles (IOP Publishing, London, 1988).

${ }^{37}$ T. Takagahara, Phys. Rev. B 31, 6552 (1985).

${ }^{38}$ N. N. Zinov'ev, L. P. Ivanov, V. I. Kozub, and I. D. Yaroshetskii, Sov. Phys. JETP 57, 1027 (1983).

${ }^{39}$ J. P. Wolfe, J. Lumin. 30, 82 (1985).

${ }^{40}$ A. E. Bulatov and S. .G. Tikhopdeev, Phys. Rev. B 46, 15058 (1992).

${ }^{41}$ M. T. Ramsbey, I. Szafranek, G. Stillman, and J. P. Wolfe, Phys. Rev. B 49, 16427 (1994).

${ }^{42}$ Tsuneya Ando, Alan B. Fowler, and Frank Stern, Rev. of Mod. Phys. 54, 437 (1982).

${ }^{43}$ A. Gold, Phys. Rev. B 38, 10798 (1988).

${ }^{44}$ H. Sakaki, T. Noda, K. Hirakawa, M. Tanaka, and T. Matsusue, Appl. Phys. Lett. 51, 1934 (1987).

${ }^{45}$ Yutaka Takahashi, Yoshimine Kato, Satoru Kano, Susumu Fukatsu, Yasuhiro Shiraki, and Ryoichi Ito, J. Appl. Phys. 76, 2299 (1994).

${ }^{46}$ J. Christen, D. Bimberg, A. Steckenborn, and G. Weimann, Appl. Phys. Lett. 44, 84 (1984). 
${ }^{47}$ M. Zachau, J. A. Kash, and W. T. Masselink, Phys. Rev. B 44, 8403 (1991).

${ }^{48}$ A. Gustafsson and L. Samuelson, Phys. Rev. B 50, 11827 (1994).

${ }^{49}$ T. C. Damen, Jagdeep Shah, D. Y. Oberli, D. S. Chemla, J. E. Cunningham, and J. M. Kuo, Phys. Rev. B 42, 7434 (1990).

${ }^{50}$ R. Strobel, R. Eccleston, J. Kuhl, and K. Köhler, Phys. Rev. B 43, 12564 (1991).

${ }^{51}$ P. W. M. Blom, P. J. van Hall, C. Smit, J. P. Cuypers, and J. H. Wolter, Phys. Rev. Lett. 71, 3878 (1993).

${ }^{52}$ T. Amand, B. Dareys, B. Baylac, X. Marie, J. Barrau, M. Brousseau, D. J. Dunstan, and R. Planel, Phys. Rev. B 50, 11624 (1994).

${ }^{53}$ Yutaka Takahashi, Satoru S. Kano, Koji Muraki, Susumu Fukatsu, Yasuhiro Shiraki, and Ryoichi Ito, Appl. Phys. Lett. 64, 1845 (1994). 


\section{FIGURES}

FIG. 1. The diagrams calculated in the exciton-electron ((a), (b)) and in the exciton-exciton ((c)-(f)) scatterings.

FIG. 2. The comparison of electron-heavy hole, exciton-heavy hole, and exciton-exciton scattering rates plotted against momentum transfer $q$.

FIG. 3. Schematic diagram of the simulation area. The initial excitons are generated in the Gaussian shape with FWHM $6 \mu \mathrm{m}$. They gradually spread out to the surrounding area with the density distribution described by the solid line. The simulation area (radius $10 \mu \mathrm{m}$ ) is divided into concentric circular regions with $1 \mu \mathrm{m}$ step, and the average carrier density at each region is used in the evaluation of carrier scatterings.

FIG. 4. The numbers of scattering events per particle per second for electrons (upper) and excitons (lower) with the initial excitation energy $10 \mathrm{meV}$ and at $5 \mathrm{~K}$. Notice that in the lower case the data are plotted in the logarithmic scale.

FIG. 5. The time evolution of the momentum distribution functions for (a) electrons and (b) excitons, from 0 ps (near side) - 15 ps (far side) with the time step 1 ps. The initial excitation energy is $10 \mathrm{meV}$ and the lattice temperature is $5 \mathrm{~K}$.

FIG. 6. The momentum distribution functions of excitons from 0 ps (near side) to 500 ps (far side) with $25 \mathrm{ps}$ step. The initial momentum distribution is in the Bose function with $T_{e x}=30 \mathrm{~K}$ and the lattice temperature at $5 \mathrm{~K}$.

FIG. 7. The FWHM of the exciton spatial distributions from 0 to 500 ps with the initial exciton temperatures changed as a parameter. The lattice temperature is fixed at $5 \mathrm{~K}$. The FWHM is obtained by fitting the Gaussian function to the spatial exciton distributions calculated in the simulations. The FWHM of the simple diffusion model with $D=30 \mathrm{~cm}^{2} \mathrm{~s}^{-1}$ is also plotted for reference. 
FIG. 8. The FWHM of the exciton spatial distributions at the lattice temperature $30 \mathrm{~K}$ with $T_{e x}=30 \mathrm{~K}$ (upper) and $5 \mathrm{~K}$ (lower).

FIG. 9. The FWHM of the exciton spatial distributions at different interface roughness parameters: the correlation length $\Lambda$ dependence (upper) and the width fluctuation $\Delta$ dependence (lower). 